\title{
Examining parent and child agreement in the diagnosis of adolescent depression
}

Article

Accepted Version

Orchard, F., Pass, L., Cocks, L., Chessell, C. and Reynolds, S. (2019) Examining parent and child agreement in the diagnosis of adolescent depression. Child and Adolescent Mental Health, 24 (4). pp. 338-344. ISSN 1475-3588 doi: https://doi.org/10.1111/camh.12348 Available at https://centaur.reading.ac.uk/86307/

It is advisable to refer to the publisher's version if you intend to cite from the work. See Guidance on citing.

To link to this article DOI: http://dx.doi.org/10.1111/camh.12348

Publisher: Wiley

All outputs in CentAUR are protected by Intellectual Property Rights law, including copyright law. Copyright and IPR is retained by the creators or other copyright holders. Terms and conditions for use of this material are defined in the End User Agreement.

\section{www.reading.ac.uk/centaur}

\section{CentAUR}

Central Archive at the University of Reading 
Reading's research outputs online 


\begin{abstract}
Background: The diagnosis of depression in adolescents relies on identifying the presence of specific core and additional symptoms. Symptoms can be identified using structured or unstructured interviews and a range of questionnaire measures, which are completed by the young person and by a parent or carer. The aim of this research was to examine the inter- and intra-rater reliability of parent report and adolescent self-report of depression symptoms. Method: In a sample of parent-child dyads, where young people aged 13-17 were referred to a mental health service for depression, we examined adolescents' $(n=46)$ and parents' $(n=$ 46) independent responses to the Schedule for Affective Disorders and Schizophrenia in School-Age Children (Kaufman et al., 1997) and the Mood and Feelings Questionnaire (Costello \& Angold, 1988).

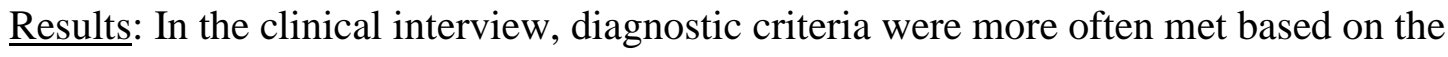
adolescent's report, and adolescents endorsed more symptoms of depression than their parents. Tentative results also suggest that parent-child agreement about specific symptoms was low. Comparing different measures of depression revealed that adolescent report on the questionnaire and interview was significantly correlated. However, there was no significant correlation between parent questionnaire and interview report.
\end{abstract}

Conclusion: These results suggest that relying solely on parents to identify depression in their children may result in young people with depression being missed and therefore untreated. Young people themselves should be encouraged and enabled to recognise the symptoms of depression, and have an established pathway to services that offer assessment and treatment. 


\section{$\underline{\text { Key Practitioner Message }}$}

- Diagnosis of depression in adolescents requires the identification of specific symptoms, and can be identified using interviews or questionnaires

- Previous research has suggested that parents and young people provide differing reports regarding symptoms of adolescent depression

- Results indicated that diagnostic criteria were more often met based on young person report, and that parents reported significantly less symptoms

- Parent-child agreement about specific symptoms was found to be low

- Assessment of adolescent depression should not rely solely on parental report

- Young people should be encouraged and enabled to recognise symptoms of depression, and be able to access mental health services.

Keywords: major depressive disorder, adolescence, symptomatology, assessment 
Adolescence is a critical period for the onset of mood disorders (Kessler et al., 2007; Merikangas, Nakamura, \& Kessler, 2009). Recent prevalence rates indicate that, at any one time, $2.7 \%$ of adolescents are experiencing depression (Polanczyk, Salum, Sugaya, Caye \& Rohde, 2015), with up to $20 \%$ of adolescents experiencing at least one depressive episode before entering adulthood (Lewinsohn, Hops, Roberts, Seeley, \& Andrews, 1993; Thapar, Collishaw, Pine, \& Thapar, 2012). Depression in adolescence is also associated with relapse later in life (Curry et al., 2011), social and educational impairment (Fergusson \& Woodward, 2002; Fletcher, 2008), physical health difficulties (Thapar et al., 2012), and represents a major risk factor for suicide (Hawton, Saunders, \& O'Connor, 2012), the leading cause of death in this age group (Windfuhr et al., 2008). Thus, it is crucial to assess depression reliably so that adolescents can access appropriate support.

The Diagnostic and Statistical Manual of Mental Disorders (DSM), the most widely used diagnostic category system, defines a diagnosis of depression in adolescents as requiring that the young person experiences at least five of nine possible symptoms, for at least two weeks, and that their functioning is impaired (DSM-5; APA, 2013). The two main approaches to assessing depression in young people are the use of interviews and rating scales. In a review of the evidence base of assessment tools for depression in children and adolescents, Klein et al. (2005) highlighted that the K-SADS is the most widely used semistructured interview, with the least structure, and requirement for greatest amount of clinical training and experience. They reported good inter-rater reliability and convergent validity and recommend that use of the K-SADS is supplemented by the need to use a range of informants as well as the interviewer's clinical judgment. Of the questionnaire scales reviewed, Klein et al. (2005) recommended the Mood and Feelings Questionnaire (MFQ; Costello \& Angold, 1988) claiming it has optimal power to discriminate between depressed and non-depressed young people and good internal consistency, test-retest reliability, and convergent validity. A 
more recent review of depression measures in children and adolescents endorsed the use of the MFQ for treatment monitoring due to the psychometric properties, evidence base and ease of incorporating into clinical practice (Simmons, Wilkinson, \& Dubicka, 2015), and existing UK guidelines for assessing and monitoring depression in young people also recommend use of the MFQ and K-SADS (National Institute for Health and Care Excellence [NICE], 2019).

Rating scales are typically used to assess symptom severity but should not be used to make a diagnosis alone (Fisher et al., 2015). Central to best practice in the assessment of adolescent mental health, is the inclusion of multiple informants' reports (De Los Reyes, Thomas, Goodman, \& Kundey, 2013), to provide a comprehensive view of the adolescents' presenting problem (Kim, Chan, McCauley, \& Vander Stoep, 2016). Although the use of multiple informants' reports is recommended, there are often different perspectives on adolescents' mental health conditions (Kim et al., 2016). In a meta-analysis, De Los Reyes et al. (2015) examined the correspondence between different informant reports of youth internalising and externalising problems. They reported only moderate levels of agreement between parents and children across clinical and non-clinical populations. However, they proposed that future work needed to focus on testing the validity of different informant assessments in the context of mood and anxiety disorders, and across developmental periods other than preschool and early adolescence.

Research which has specifically addressed the difference between parent and child self-report of depression has consistently found that parents report lower levels of depression symptoms than their child's report in both community (Eg, Bilenberg, Costello, \& Wesselhoeft, 2018) and clinical populations (Orchard, Pass, Marshall, \& Reynolds, 2017). Furthermore, Orchard et al. (2017) also identified that there was no significant pairwise correlation between parent and adolescent reports of symptom severity. There are many 
factors that are likely to moderate cross-informant agreement, including parental depression (Chi \& Hinshaw, 2002; Najman, Andersen, Bor, O'Callaghan, \& Williams, 2000), the degree to which symptoms can be directly observed, and the extent that they are present at home, rather than at school or other settings (Comer \& Kendall, 2004).

In a semi-structured clinical interview, assessors are trained to elicit specific information relative to diagnostic thresholds for individual symptoms from both parent and child informants, and it is therefore plausible that data obtained in this way may not be subject to the same level of disagreement between young people and their caregivers that is seen on a questionnaire. Angold et al. (1987) elicited reports of depressive symptomology from 214 parents and their 181 offspring (aged 7-25 years) on the Schedule for Affective Disorders and Schizophrenia for School-Aged Children, Epidemiologic Version (K-SADS-E; Orvaschel, Puig-Antich, Chambers, Tabrizi, \& Johnson, 1982). Participants were young people who were considered at high or low risk of depression, based on parental psychiatric history but who were not currently in contact with mental health services. Parents consistently reported fewer depressive symptoms than their offspring. There was significant agreement between informants on some symptoms, particularly suicidality, however overall agreement on individual symptoms was low.

Multi-informant disagreement may reflect, in part, differences in the contexts and perspective from which informants rate the young person's symptoms (e.g. De Los Reyes et al., 2015; De Los Reyes et al., 2013). However, disagreement about symptoms between parents and adolescents that are seeking help for the child's depression would have major implications for accessing treatment. Initial contact with general practitioners and with specialist child and mental health services is typically made by parents, and the initial assessment or screening of the child's difficulties is therefore often conducted with the parent only. If parents are reporting fewer of their child's symptoms, this could have implications 
for whether a child is considered suitable or eligible for a given service. Therefore it is important to know the extent to which parent report and child self-report align when planning service referral pathways and eligibility criteria.

In this study we aim to assess the concordance between parents and adolescent offspring on both a semi-structured diagnostic interview and a standardised questionnaire measure of depressive symptoms, in a sample where the adolescents were referred to a specialist mental health service with symptoms of depression. On the basis of previous research three research questions were examined. The first research question aimed to replicate previous research findings, and the additional exploratory research questions were formulated to extend this line of work.

1. Are diagnostic criteria for depression more often met on the basis of adolescent or parent report?

2. Do adolescents and parents agree about the presence of specific symptoms of depression on a semi-structured diagnostic interview (K-SADS)?

3. What is the level of agreement within individuals on the presence of specific depression symptoms using alternative measures of depression (MFQ vs K-SADS)?

\section{Methods}

\section{Participants}

Participants were a subset of those who took part in previous research (Orchard et al., 2017). Adolescents and their parents were referred by primary and secondary care services to a UK child and adolescent mental health service which assesses and treats children and adolescents with anxiety and depression. The subset of participants in the present study included families who consented to audio recordings of both child and parent diagnostic assessment. 
Fifty-two dyads of parents and adolescents (aged 13-17 years) were eligible to take part in the study. Six pairs had more than $25 \%$ of data missing on the interview measure and were excluded from analysis, leaving a final sample of forty-six parent-child dyads (i.e. a total sample of $\mathrm{N}=92$ ).

The mean age of the adolescents was 15.64 years $(S D=1.16)$ and the majority were female (82.6\%). Participants were mainly White British $(87.5 \%, n=40)$, with other ethnicities including white and black Caribbean, Asian, Indian and Pakistani. Occupational backgrounds of the parents were dichotomised according to Office of National Statistics Socioeconomic classification system as 'professional' or 'non-professional' with most families classified as 'professional' $(74.3 \%, n=35)$. Biological mothers $(78 \%)$ were the main primary caregivers assessed, with biological fathers (14\%) and adoptive or step-parents (8\%) as the remaining informants.

Twenty-seven of the adolescents in the current sample met diagnostic criteria for a DSM-IV diagnosis (see Table 1). Of these 27 adolescents, 10 met criteria for one comorbid disorder. No adolescent presented with more than one comorbid disorder.

\section{Measures}

Questionnaire measure. The Mood and Feelings Questionnaire (MFQ; Costello \& Angold, 1988) is a 33 item self-report scale of depressive symptoms for adolescents which has good psychometric properties (Burleson Daviss et al., 2006). The scale has been reported to have good internal consistency and test-retest reliability (Angold et al., 1995; Wood, Kroll, Moore, \& Harrington, 1995), and good convergent validity (Angold et al., 1995; Thapar \& McGuffin, 1998; Wood et al., 1995). It has also bee identified as being able to discriminate those with and without diagnoses of depression (Kent, Vostanis, \& Feehan, 1997; Thapar \& McGuffin, 1998) with evidence of sensitivity to change in clinical trials (Brooks \& Kutcher, 2001; Goodyer et al., 2017). 
This study included adolescent (MFQ-C) and parent (MFQ-P) versions of the questionnaire. These versions include the same questions but reworded for parent or child, for example, 'I felt miserable or unhappy' (MFQ-C) and 'S/he felt miserable or unhappy' (MFQP. Each symptom is rated on a 3-point scale from 0 (not true) to 2 (true). Internal consistency for the MFQ was very high (MFQ-C $\alpha=.91 ;$ MFQ-P $\alpha=.95$ ).

Diagnostic interview. Adolescents referred to the clinic were assigned diagnoses based on the Kiddie Schedule of Affective Disorders and Schizophrenia (K-SADS), a semistructured diagnostic interview for DSM-IV affective disorders and schizophrenia (Kaufman et al., 1997). The K-SADS has well-established psychometric properties, including good inter-rater reliability and convergent validity (Ambrosini \& Dixon, 2000; Kaufman et al., 1997). To meet criteria for a diagnosis of depression using the K-SADS, adolescents are required to meet symptom thresholds for at least 5 symptoms of depression, which must also include at least one of the core symptoms of depression; anhedonia, depressed mood or irritability (in line with DSM-5 criteria; APA, 2013). See Orchard et al. (2017) for details of the method of assigning diagnoses, and the process of training and reliability in this sample.

\section{[INSERT TABLE 1]}

\section{Procedure}

Adolescents aged 16-17 years provided informed consent for themselves, while adolescents aged 13-15 years required consent from their parent as well as providing assent themselves. Parents also provided written consent for themselves taking part. Parents and adolescents attended a clinical assessment where they took part in a diagnostic interview and completed standardised symptom questionnaires. The symptom measure and diagnostic interview were completed on the same day by both the adolescent and their parent independently. K-SADS interviews with parents and adolescents were conducted separately, 
and interviews were audio-taped and detailed assessment notes taken.

For the purpose of this study, each interview recording $(\mathrm{N}=92)$ was coded according to K-SADS diagnostic criteria by two research assistants (LC \& CC) who were trained using the same procedure as the original assessors. This was to determine the separate parent and child outcomes on the K-SADS assessment (this could not be identified from the original clinical assessment outcomes, as symptoms were recorded as present/absent based on the combination of parent and child report). To confirm reliability, $10 \%$ of the samples were rated independently by both coders; inter-rater reliability was high $(\kappa=.81$; Landis \& Koch, 1977). In the case of disagreements coding was discussed and agreed by consensus.

\section{Data preparation}

The total number of symptoms endorsed by each informant was calculated, and two separate diagnostic profiles for the adolescent were determined based on (1) self-reported adolescent diagnosis, and (2) parent-reported adolescent diagnosis.

As is standard, depressed mood and irritability were assessed and scored separately, however, for diagnostic purposes they are considered to be one overall symptom. Therefore, where research questions address diagnoses, depressed mood and irritability were counted as one of the minimum five out of nine symptoms (in line with DSM-IV). Where research questions address symptom-level questions, depressed mood and irritability are treated as separate symptoms, resulting in a maximum of 10 possible reported symptoms.

\section{Preliminary data analysis}

\section{Results}

Continuous data were screened in relation to the assumptions of parametric tests (Tabachnick \& Fidell, 2007). Where assumptions were violated, confirmatory analyses were conducted by running analyses with 1000 bootstrap samples or non-parametric alternatives. All results were consistent, suggesting that the original analyses were robust to the violations of assumptions, so results based on the original (non-bootstrapped) analyses are presented for 
simplicity. Categorical data (symptom present or absent) were screened against the assumptions of chi square and kappa. Requirements of mutual exclusivity were met, and when clustered there were no expected frequencies fewer than 5 in individual symptom analyses.

\section{Identification of depression diagnosis using separate parent and child report}

To address this question we used a range of approaches. First we used a chi-square to assess the association between depression diagnosis (diagnosis given vs diagnosis not given) based on interviews with parents and with adolescents. Second we used an independent samples t-test to compare the number of depression symptoms endorsed by parents and by adolescents and examined the correlation between the number of depression symptoms endorsed by parents and their offspring using paired correlations.

[INSERT TABLE 2]

Table 2 shows that a depression diagnosis was more often given on the basis of adolescent report compared to parent report. The chi square analysis was significant, $X^{2}(1)=$ $12.36, p<.001$. Of the 20 adolescents that met a depression diagnosis according to selfreport, $80 \%(n=16)$ originally met for a depression diagnosis when information from both informants was discussed and symptoms agreed in clinical supervision.

Parents also reported significantly fewer adolescent symptoms of depression on the K-SADS $(M=1.98, S D=1.92)$ than adolescents themselves $(M=4.13, S D=2.38), t(90)=$ $4.54, p<.001$. There was a significant positive correlation between the number of depression symptoms endorsed by parents and adolescents $(r=.38, p=.01)$.

Parent-child agreement about the presence of specific symptoms of depression 
Firstly, to examine whether parents (as a group) endorsed similar symptoms to adolescents (as a group), we conducted individual chi square analyses on the overall frequency with which each of the 10 depression symptoms were endorsed on the K-SADS. Table 3 shows the overall frequency of symptoms endorsed by adolescents and parents on the K-SADS. For parents and adolescents, the most commonly endorsed symptom was negative self-perceptions. Parents were less likely to endorse every symptom than young people. After correcting for multiple testing, parents were significantly less likely than their child to endorse suicidal thoughts, sleep disturbances and fatigue. Cramer's V effect sizes also demonstrate medium effects for suicidal thoughts, sleep disturbances, fatigue and depressed mood.

\section{[INSERT TABLE 3]}

Secondly, to examine whether parents and their offspring agreed about the presence (or absence) of depression symptoms experienced by the adolescent in that dyad, we calculated inter-rater reliability within the dyads (Cohen's kappa) on each symptom of depression. Within each dyad, agreement on the presence or absence between parent and their offspring ranged from $54-78 \%$. The kappa statistic indicates that most of the symptoms would be considered to show 'none to slight' agreement, with three symptoms showing 'fair' agreement (irritability, sleep disturbances and eating disturbances) (Cohen, 1968).

[INSERT TABLE 4]

\section{Agreement within individuals on the presence of specific depression symptoms using alternative measures of depression}


We conducted pairwise correlations between MFQ total score and the number of symptoms endorsed on the K-SADS. For adolescent report, there was a significant positive relationship between the number of K-SADS symptoms $(M=4.13, S D=2.38)$ and MFQ score $(M=38.11, S D=11.98), r=.51, p=.001$. There was no significant correlation between the number of K-SADS symptoms $(M=1.98, S D=1.92)$ and MFQ score $(M=$ 27.44, $S D=14.70)$ for parent report, $r=.11, p=.54$.

\section{Discussion}

This is the first study to examine in detail parent and adolescent reports of adolescent depression symptoms using both a semi-structured clinical interview (K-SADS) and a selfreport symptom questionnaire (MFQ). When reporting on depression symptoms experienced by their own child, parents identified significantly fewer symptoms than their child and typically did not identify the same symptoms as the adolescent. Adolescents consistently endorsed more symptoms than their parents on the MFQ and on the K-SADS. Therefore it is not surprising that diagnoses were more often met on the basis of the adolescent's report than on the basis of a report by their parent. Agreement between the parent and adolescent was also low for most of the individual symptoms, with just three symptoms demonstrating some agreement. Also of concern was the low level of agreement between the MFQ and the KSADS when the same informant answered questions about the same symptoms, on the same day. Although a significant positive relationship was identified between measures for adolescent report, there was no significant correlation between parents' reports on the same instruments.

Previous research has suggested that parents endorse fewer symptoms of their child's depression than adolescents themselves, on both the MFQ (Orchard et al., 2017), and the KSADS (Angold et al., 1987). Orchard et al. (2017) also reported that there was no significant pairwise correlation between parent and adolescent reports of adolescent depression on the 
MFQ. The current study replicated the finding that parents endorse fewer symptoms on the K-SADS than adolescents. However, in contrast to research by Orchard et al. (2017) using a questionnaire measure, parent and child report on the number of K-SADS symptoms was significantly and positively correlated.

In terms of agreement on individual symptoms, previous work by Angold et al. (1987) reported high agreement between parents and adolescents regarding suicidal ideation; in contrast our results indicated that agreement between parent and adolescent's reports of suicidal ideation was low. This discrepancy may reflect differences in sampling across the two studies. Angold et al. (1987), recruited participants aged 7-25 years who were at elevated risk of depression because of a family history of depression, but who were not currently seeking help. The participants recruited in the current study were aged 13-17 years, were referred to a mental health service, and overall levels of distress, including suicidal ideation, were higher than in Angold et al. (1987). There may also be important differences over time - e.g. young people may have become be more prepared to report suicidal ideation during the intervening three decades. As might be expected, on the K-SADS interview data, parent-child agreement was highest for external behaviours i.e. irritability, sleep disturbances and eating disturbances. This is consistent with research drawing on broader health related assessments in children. A recent systematic review reported that agreement was generally only poor to moderate, but with several studies reporting more agreement on observable or external domains (Hemmingsson, Ólafsdóttir, \& Egilson, 2017).

A strength of the present study was the recruitment of a sample of adolescents and parents from consecutive referrals to an NHS Child and Adolescent Mental Health service in the UK. Thus, this sample is likely to be typical of the population of referred young people in the UK where it would be expected that some adolescents referred for suspected depression would meet diagnostic criteria, but many may not meet diagnostic criteria for depression and 
others may not meet criteria for any other emotional disorder. The semi-structured interview method used, the K-SADS, is considered the 'gold standard' method of assessing depression in young people and our assessors were trained, reliable and blind to the diagnostic decisions made in the clinic.

The nature of the help-seeking population in the present study may have implications for the findings. Given that parents are typically gate-keepers into mental health services, the present sample may be more likely to have higher agreement than non-help-seeking families where parents might be less aware of the difficulties that their children are experiencing. As such it is possible that the rates of agreement, while being low, may be higher than might be seen across the general population. Future research would benefit from exploring these research questions in different samples of young people and their parents.

The analysis of this study focussed on agreement between depression symptoms, but it is possible that parents have a better understanding of functioning and impairment in their children. Future work may also benefit from measuring concordance on measures of impact and interference, especially given the emerging importance of these factors in identifying diagnostic status in young people (e.g. Evans, Thirlwall, Cooper, \& Creswell, 2017). Furthermore, the sample size was modest and thus greater precision would be possible with a larger sample of participants. This study was not designed to explore factors that may explain disagreement between parents and children, for example, parental psychopathology, age of child, context-specific knowledge.

The results of this study have important implications for research and clinical work. Prompt identification of depression in adolescents is important to ensure access to evidencebased treatment and appropriate support. The lack of concordance between parents and their adolescent offspring is important and concerning. Initial contact with specialist child and adolescent mental health services is typically made by parents rather than by young people 
themselves. Given that parents reported significantly fewer symptoms of depression experienced by their son or daughter, and were significantly less likely to report symptoms required for a diagnosis of depression, this reliance on parents to identify depression in their children suggests that many young people with depression may go un-identified and therefore untreated. One obvious implication for service design and delivery systems is that young people themselves should be encouraged and enabled to recognise the symptoms of depression and other common mental health problems and have ready and direct access to services that offer assessment and treatment.

Furthermore, the lack of association between parent interview and questionnaire measures may suggest that services could be more strategic in the data that are collected from parents. The descriptive statistics examining these measures for parents show that mean scores for the MFQ, and are indicative of clinical levels of depression, i.e. >27 (Burleson Daviss et al., 2006). However, the mean number of depression symptoms endorsed on the KSADS is much lower than required for a diagnosis. This may suggest that if services were to retain one measure from parents, they would benefit more from collecting questionnaire measures of symptomatology. However, it could also be argued that by exploring parental knowledge of individual symptoms in an interview, that this may reveal where gaps in knowledge exist and may help to open up a conversation with parents about areas of behaviour that are important to be monitor. This may prove particularly important with helping parents better monitor suicidal ideation and actions.

Guidelines for the identification of depression indicate that a diagnostic interview is preferable (NICE, 2019). The results of this study suggest that parent report does not contribute significantly to the diagnoses that are made, mainly because parents report consistently fewer of their children's symptoms. Therefore whilst a holistic assessment may require parental report to obtain a family history, assess current risk and protective factors, 
and develop a formulation on which to base a treatment plan, detailed parental report may be less informative in making a diagnosis of adolescent depression. Given the results reported here and in the wider literature, as well as the implications for young people's access to treatment for depression, this is an issue that warrants further clinical and empirical investigation. 


\section{Acknowledgements}

The research was funded by a PhD studentship at the University of Reading. The authors have declared that they have no competing or potential conflicts of interest. Study design was carried out by FO, SR and LP, data collection was completed out by FO, coding was carried out by LC and CC, data analysis was conducted by FO, LC and CC, and all authors were involved in drafting and editing the manuscript.

The authors would like to thank the participating families and the staff at the Anxiety and Depression Pathway at the University of Reading, in particular Rachael Adams, Sarah Armitage, Sophie Boothe, Kirsten Corden, Sue Cruddace, Jeni Fisk, Poppy Jones, Tamsin Marshall, Ambreen Masih, Emily Nobes, Louise Noble, Alice Parker, Jasmine Raw, Hannah Rogers, Holly Tricker, Nick White and Lucy Willetts for their help collecting and coding data. 


\section{References}

Ambrosini, P., \& Dixon, J. (2000). Schedule for affective disorders \& schizophrenia for school age children (6-18 years)-Kiddie-SADS (KSADS)(present state and lifetime version) K-SADS-IVR (Revision of K-SADS-IIIR). Unpublished manuscript. Eastern Pennsylvania Psychiatric Institute, Philadelphia, PA.

Angold, A., Costello, E. J., Messer, S. C., Pickles, A., Winder, F., \& Silver, D. (1995). Development of a short questionnaire for use in epidemiological studies of depression in children and adolescents. International Journal of Methods in Psychiatric Research, 5, 237-249.

Angold, A., Weissman, M. M., John, K., Merikancas, K. R., Prusoff, B. A., Wickramaratne, P., . . . Warner, V. (1987). Parent and child reports of depressive symptoms in children at low and high risk of depression. Journal of Child Psychology and Psychiatry, 28(6), 901-915.

APA. (2013). Diagnostic and statistical manual of mental disorders (DSM-5). Arlington: American Psychiatric Publishing.

Brooks, S. J., \& Kutcher, S. (2001). Diagnosis and measurement of adolescent depression: a review of commonly utilized instruments. Journal of child and adolescent psychopharmacology, 11(4), 341-376.

Burleson Daviss, W., Birmaher, B., Melhem, N. A., Axelson, D. A., Michaels, S. M., \& Brent, D. A. (2006). Criterion validity of the Mood and Feelings Questionnaire for depressive episodes in clinic and non-clinic subjects. Journal of Child Psychology and Psychiatry, 47(9), 927-934.

Chi, T. C., \& Hinshaw, S. P. (2002). Mother-child relationships of children with ADHD: The role of maternal depressive symptoms and depression-related distortions. Journal of Abnormal Child Psychology, 30(4), 387-400.

Cohen, J. (1968). Weighted kappa: Nominal scale agreement provision for scaled disagreement or partial credit. Psychological Bulletin, 70(4), 213.

Comer, J. S., \& Kendall, P. C. (2004). A symptom-level examination of parent-child agreement in the diagnosis of anxious youths. Journal of the American Academy of Child \& Adolescent Psychiatry, 43(7), 878-886.

Costello, J. E., \& Angold, A. (1988). Scales to assess child and adolescent depression - checklists, screens and nets. Journal of the American Academy of Child and Adolescent Psychiatry, 27(6), 726-737.

Curry, J., Silva, S., Rohde, P., Ginsburg, G., Kratochvil, C., Simons, A., . . Mayes, T. (2011). Recovery and recurrence following treatment for adolescent major depression. Archives of General Psychiatry, 68(3), 263-269.

De Los Reyes, A., Augenstein, T. M., Wang, M., Thomas, S. A., Drabick, D. A., Burgers, D. E., \& Rabinowitz, J. (2015). The validity of the multi-informant approach to assessing child and adolescent mental health: American Psychological Association.

De Los Reyes, A., Thomas, S. A., Goodman, K. L., \& Kundey, S. M. (2013). Principles underlying the use of multiple informants' reports. Annual Review of Clinical Psychology, 9, 123-149.

Eg, J., Bilenberg, N., Costello, E. J., \& Wesselhoeft, R. (2018). Self-and parent-reported depressive symptoms rated by the Mood and Feelings Questionnaire. Psychiatry Research, 268, 419425.

Evans, R., Thirlwall, K., Cooper, P., \& Creswell, C. (2017). Using symptom and interference questionnaires to identify recovery among children with anxiety disorders. Psychological assessment, 29(7), 835.

Fergusson, D. M., \& Woodward, L. J. (2002). Mental health, educational, and social role outcomes of adolescents with depression. Archives of General Psychiatry, 59(3), 225-231.

Fisher, P. W., Chin, E. M., Vidair, H. B., Thapar, A., Pine, D. S., Leckman, J. F., . . Taylor, E. (2015). Use of structured interviews, rating scales, and observational methods in clinical settings Rutter's Child and Adolescent Psychiatry (pp. 419-435): John Wiley \& Sons, Ltd. 
Fletcher, J. M. (2008). Adolescent depression: diagnosis, treatment, and educational attainment. Health economics, 17(11), 1215-1235.

Gammon, G. D., John, K., Rothblum, E. D., Mullen, K., Tischler, G. L., \& Weissman, M. M. (1983). Use of a structured diagnostic interview to identify bipolar disorder in adolescent inpatients: Frequency and manifestations of the disorder. Am J Psychiatry, 140(5), 543-547.

Goodyer, I. M., Reynolds, S., Barrett, B., Byford, S., Dubicka, B., Hill, J., . . Roberts, C. (2017). Cognitive-behavioural therapy and short-term psychoanalytic psychotherapy versus brief psychosocial intervention in adolescents with unipolar major depression (IMPACT): a multicentre, pragmatic, observer-blind, randomised controlled trial. Health Technology Assessment (Winchester, England), 21(12), 1.

Hawton, K., Saunders, K. E., \& O'Connor, R. C. (2012). Self-harm and suicide in adolescents. The Lancet, 379(9834), 2373-2382.

Hemmingsson, H., Ólafsdóttir, L. B., \& Egilson, S. T. (2017). Agreements and disagreements between children and their parents in health-related assessments. Disability and rehabilitation, 39(11), 1059-1072.

Kaufman, J., Birmaher, B., Brent, D., Rao, U., Flynn, C., Moreci, P., . . Ryan, N. (1997). Schedule for affective disorders and schizophrenia for school-age children-present and lifetime version (K-SADS-PL): initial reliability and validity data. Journal of the American Academy of Child \& Adolescent Psychiatry, 36(7), 980-988.

Kent, L., Vostanis, P., \& Feehan, C. (1997). Detection of major and minor depression in children and adolescents: evaluation of the Mood and Feelings Questionnaire. Journal of Child Psychology and Psychiatry, 38(5), 565-573.

Kessler, R. C., Angermeyer, M., Anthony, J. C., De Graaf, R., Demyttenaere, K., Gasquet, I., . . . Haro, J. M. (2007). Lifetime prevalence and age-of-onset distributions of mental disorders in the World Health Organization's World Mental Health Survey Initiative. World psychiatry, 6(3), 168.

Kim, J., Chan, Y.-F., McCauley, E., \& Vander Stoep, A. (2016). Parent-Child Discrepancies in Reporting of Child Depression in Ethnic Groups. The Journal for Nurse Practitioners, 12(6), 374-380.

Klein, D. N., Dougherty, L. R., \& Olino, T. M. (2005). Toward guidelines for evidence-based assessment of depression in children and adolescents. Journal of Clinical Child and Adolescent Psychology, 34(3), 412-432.

Landis, J. R., \& Koch, G. G. (1977). The measurement of observer agreement for categorical data. biometrics, 159-174.

Lewinsohn, P. M., Hops, H., Roberts, R. E., Seeley, J. R., \& Andrews, J. A. (1993). Adolescent psychopathology: I. Prevalence and incidence of depression and other DSM-III-R disorders in high school students. Journal of Abnormal Psychology, 102(1), 133.

Merikangas, K. R., Nakamura, E. F., \& Kessler, R. C. (2009). Epidemiology of mental disorders in children and adolescents. Dialogues in clinical neuroscience, 11(1), 7.

Najman, J., Andersen, M., Bor, W., O'Callaghan, M., \& Williams, G. (2000). Postnatal depressionmyth and reality: maternal depression before and after the birth of a child. Social psychiatry and psychiatric epidemiology, 35(1), 19-27.

National Institute for Health and Care Excellence. (2013). Depression in children and young people: identification and management (NICE Guideline No. 134). Retrieved from https://www.nice.org.uk/guidance/ng134

Orchard, F., Pass, L., Marshall, T., \& Reynolds, S. (2017). Clinical characteristics of adolescents referred for treatment of depressive disorders. Child and Adolescent Mental Health, 22(2), 61-68.

Orvaschel, H., Puig-Antich, J., Chambers, W., Tabrizi, M. A., \& Johnson, R. (1982). Retrospective assessment of prepubertal major depression with the Kiddie-SADS-E. Journal of the American Academy of Child Psychiatry, 21(4), 392-397. 
Polanczyk, G. V., Salum, G. A., Sugaya, L. S., Caye, A., \& Rohde, L. A. (2015). Annual Research Review: A meta-analysis of the worldwide prevalence of mental disorders in children and adolescents. Journal of Child Psychology and Psychiatry, 56(3), 345-365. Simmons, M., Wilkinson, P., \& Dubicka, B. (2015). Measurement Issues: Depression measures in children and adolescents. Child and Adolescent Mental Health, 20(4), 230-241.

Tabachnick, B. G., \& Fidell, L. S. (2007). Experimental designs using ANOVA: Thomson/Brooks/Cole. Thapar, A., Collishaw, S., Pine, D. S., \& Thapar, A. K. (2012). Depression in adolescence. The Lancet, 379(9820), 1056-1067.

Thapar, A., \& McGuffin, P. (1998). Validity of the shortened Mood and Feelings Questionnaire in a community sample of children and adolescents: a preliminary research note. Psychiatry Research, 81(2), 259-268.

Windfuhr, K., While, D., Hunt, I., Turnbull, P., Lowe, R., Burns, J., . . Kapur, N. (2008). Suicide in juveniles and adolescents in the United Kingdom. Journal of Child Psychology and Psychiatry, 49(11), 1155-1165.

Wood, A., Kroll, L., Moore, A., \& Harrington, R. (1995). Properties of the Mood and Feelings Questionnaire in adolescent psychiatric outpatients: A research note. Journal of Child Psychology and Psychiatry, 36, 327-334. 
Table 1: Diagnostic characteristics of the adolescent sample $(n=46)$

Diagnosis Primary (Overall) \%

\begin{tabular}{lc}
\hline Major Depressive Disorder & $26.1(47.8)$ \\
Social Phobia & $10.9(21.7)$ \\
Generalised Anxiety Disorder & $8.7(17.4)$ \\
Obsessive Compulsive Disorder & $2.2(4.3)$ \\
Oppositional Defiant Disorder & $2.2(2.2)$ \\
MDD with Psychosis & $4.3(4.3)$ \\
ADNOS & $2.2(4.3)$ \\
Agoraphobia & $2.2(2.2)$ \\
No Axis 1 Disorder & $41.3(41.3)$
\end{tabular}

NB. ADNOS: Anxiety disorder not otherwise specified; MDD: Major Depressive Disorder. 
Table 2. Frequency table of diagnostic outcome for parent and adolescent informants $(\mathrm{N}=$ 92)

\begin{tabular}{lll}
\hline & Diagnosis & No Diagnosis \\
\hline Parent & $5(11 \%)$ & $41(89 \%)$ \\
\hline Adolescent & $20(43 \%)$ & $26(57 \%)$ \\
NB. Diagnosis determined on the basis of each individual report.
\end{tabular}


Table 3: Frequency of symptoms and association between parent and adolescent report on the K-SADS $(\mathrm{N}=92)$

\begin{tabular}{l|cccc} 
& Adolescent & Parent & & \\
Symptom & endorsement (\%) & endorsement (\%) & $X^{2}$ & $w$ \\
\hline Depressed Mood & 52.2 & 17.5 & 7.79 & 0.41 \\
Irritability & 30.4 & 26.1 & 0.21 & 0.07 \\
Anhedonia & 17.4 & 6.5 & 2.58 & 0.24 \\
Suicidal Thoughts & 39.1 & 8.7 & $11.35^{*}$ & 0.50 \\
Sleep Disturbances & 32.6 & 6.5 & $9.95^{*}$ & 0.47 \\
Fatigue & 56.5 & 21.7 & $11.68^{*}$ & 0.50 \\
Cognitive Disturbances & 45.7 & 30.4 & 2.26 & 0.22 \\
Eating Disturbances & 32.6 & 19.6 & 2.03 & 0.21 \\
Psychomotor Disturbances & 17.4 & 10.9 & 0.81 & 0.13 \\
Negative Self-Perceptions & 63.0 & 54.3 & 0.72 & 0.13 \\
\hline NB. & & & & \\
\hline
\end{tabular}

NB. * indicates significant disagreement for chi square; $p<.005$ (corrected for multiple testing) 


\begin{tabular}{l|cc} 
& \multicolumn{2}{|c}{ Informant } \\
Symptom & agreement (\%) & $\kappa$ \\
\hline Depressed Mood & 74 & .19 \\
Irritability & 76 & .36 \\
Anhedonia & 67 & .11 \\
Suicidal Thoughts & 78 & .25 \\
Sleep Disturbances & 57 & .19 \\
Fatigue & 54 & .06 \\
Cognitive Disturbances & 74 & .34 \\
Eating Disturbances & 72 & -.15 \\
Psychomotor Disturbances & 57 & .11 \\
Negative Self-Perceptions & & \\
\hline
\end{tabular}

